\title{
Modeling of Optical Gain Properties of Multiple Cations InGaAs-InAlAs Quantum-Well Intermixing
}

\author{
Michael C. Y. Chan, Y. Chan, and E. Herbert Li, Senior Member, IEEE
}

\begin{abstract}
Multiple cations intermixing in an $\operatorname{In}_{0.53} \mathbf{G a}_{0.47} \mathrm{As}-$ $\mathbf{I n}_{0.52} \mathbf{A l}_{0.48} A s$ quantum-well (QW) structure with $\mathbf{6 0}-\AA$, well width is being studied based on the expanded form of Fick's second law. Interdiffusion of the indium sublattice can result in a maximum compressive strain of $0.64 \%$ when annealing time reaches $3 \mathrm{~h}$ at $812{ }^{\circ} \mathrm{C}$. For a small interdiffuison, i.e., 1-1.5 h, the subband separation between the lowest heavy and light hole states is at its greatest. This has a major contribution to the modified band structure and averaged density of states which can result in an enhanced optical gain up to $40 \%$. This initial stage of intermixing provides the best lasing performance. For large interdiffusion, i.e., up to $6 \mathrm{~h}$, a large blue shift of the peak gain from $0.842 \mathrm{eV}(\lambda=1.47 \mu \mathrm{m})$ to $1.016 \mathrm{eV}(\lambda=1.22 \mu \mathrm{m})$ is obtained, thus giving a high tunability of the lasing wavelength.
\end{abstract}

Index Terms - Diffusion process, InAlGaAs, optical gain, quantum-well interdiffusion, quantum-well intermixing, quantum-well lasers, tunable devices.

\section{INTRODUCTION}

$\mathbf{T}$ HE $\mathrm{In}_{x} \mathrm{Ga}_{1-x} \mathrm{As}-\mathrm{In}_{0.52} \mathrm{Al}_{0.48} \mathrm{As}$ quantum-well (QW) structures fabricated on InP substrate have a remarkable potential for application in long-haul and wide-bandwidth optical communication systems. The $\operatorname{In}_{0.52} \mathrm{Al}_{0.48} \mathrm{As}$ barrier is lattice-matched to the substrate and changing the Group III sublattice composition in the InGaAs QW will give rise to different types of strain. The as-grown $\mathrm{In}_{0.53} \mathrm{Ga}_{0.47} \mathrm{As}-\mathrm{In}_{0.52} \mathrm{Al}_{0.48} \mathrm{As} \mathrm{QW}$ has a bandgap transition energy of $0.84 \mathrm{eV}$ (wavelength $\lambda=1.48 \mu \mathrm{m}$ ), by the introduction of strain through the thermally induced QW composition intermixing technique [1], a tunable operation wavelength around $1.3 \mu \mathrm{m}$ can be obtained. This material system also offers high-speed modulation which is free from chirping [2], and it therefore finds applications in electroabsorption modulators [3], [4] and electrooptic waveguide switches [5] with switching capability up to multi-gigahertz. The InGaAs-InAlAs QW has many advantages over the quaternary InGaAsP materials. While the growth of highquality quaternary materials such as InGaAsP remains a challenge, ternary InGaAs-InAlAs QW material system on InP substrate can become a promising candidate. Apart from a high material quality, good optical properties can be obtained by interdiffusion (otherwise knows as intermixing) which changes the content of In, Ga, and $\mathrm{Al}$ in the InGaAs well to a latticemismatched structure. This gives significant modification in

Manuscript received May 21, 1997; revised November 24, 1997. This paper is supported by the RGC Earmarked Grant of Hong Kong and the University of Hong Kong CRCG Research Grant.

The authors are with the Department of Electrical and Electronic Engineering, University of Hong Kong, Pokfulam Road, Hong Kong.

Publisher Item Identifier S 0018-9197(98)01764-3. the valence-band structure and hence improvement in the optical properties. The use of a strained QW is know to be very important for the development of very low threshold current diode lasers.

The post-growth process of thermally induced composition intermixing of QW's has becoming a popular technique for material tuning, which is proposed here. The band structure [6] and types of induced strain [7] intermixing process changes in the interdiffused QW (DFQW). Consequently, a joint density of states, an optical transition element, and other optical parameters will change accordingly. The interdiffusion has been studied in various material systems, including the more popular AlGaAs-GaAs [6], [8], InGaAs-GaAs [9], [10], InGaAs-InP [11], [12], and $\mathrm{Si}-\mathrm{Ge}$ [13]. Previous works have reported on the not so common systems, such as GaAsSb-GaAs [14] and $\mathrm{CdSe}-\mathrm{ZnSe}$ [15]. The interdiffusion techniques are not only applicable in the tunability of device, enhancement in operation can also be obtained. In order to modify the device performance and to achieve tunability, a well-controlled interdiffusion technique is essential. By using impurity-induced enhanced intermixing, through ion implantation [16] or diffusion [17], and alternatively by impurity-free vacancy enhanced intermixing technique [18] through a masking process, selective area intermixing can be achieved planarly. These techniques not only have provided the wavelength tunability of devices [19], but it can also improve device performance [20] and are promising technologies for the integration of photonic integrated circuits (IC's) [21].

In this paper, we present a detailed theoretical analysis of the cation interdiffused InGaAs-InAlAs QW's. This includes the confinement profiles, band structures and optical properties, such as optical gain, differential gain, and transparency carrier density. In Section II, we will present the models for three species interdiffusion, induced strain, band structure, and optical properties. In Section III, a lattice-matched asgrown structure is selected to demonstrate how the optical gain is affected by the technique of intermixing. Finally the conclusions are drawn.

\section{MODELING}

\section{A. Multiple Species Interdiffusion}

The QW studied here is InGaAs-InAlAs. In this system, multiple group-III species (In, Al, and $\mathrm{Ga}$ ) become available as the interdiffusable constituent atoms. The interdiffusion process can be modeled by the expanded form of Fick's second 
law

$$
\frac{\partial}{\partial t}\left(\begin{array}{l}
w_{1} \\
w_{2}
\end{array}\right)=\frac{\partial}{\partial z}\left[\left[\begin{array}{ll}
D_{11} & D_{12} \\
D_{21} & D_{22}
\end{array}\right] \cdot \frac{\partial}{\partial z}\left(\begin{array}{l}
w_{1} \\
w_{2}
\end{array}\right)\right]
$$

where $w_{1}=w_{1}(z, t)$ and $w_{2}=w_{2}(z, t)$ are the concentration profiles of In and $\mathrm{Ga}$, respectively, $D_{i j}(i, j=1,2)$ are the diffusion coefficients, $D_{i i}$ is the diffusion rates of species $i$, that is, $D_{11}$ and $D_{22}$ are the diffusion rates of In and $\mathrm{Ga}$, respectively, and $D_{i j}$ is the cross diffusion rate between species $i$ and $j$ such that $D_{12}$ is the diffusion rates of In-Ga and $D_{21}$ is that of Ga-In. The growth and hence the interdiffusion direction is along $z$ and the center of the QW is defined at $z=0$. The values of $D_{i j}$ and the determination of concentrations have been previously determined [22]. Applying the stoichiometry boundary condition, the concentration of $\mathrm{Al}$ is calculated by $1-w_{1}-w_{2}$.

\section{B. Strain}

Lattice mismatch between thin-well and thick-barrier QW materials can be taken up by strain and results in a pseudomorphic QW such that a uniform lattice constant can be found through out the whole structure. This tetragonal deformation results in strain is perpendicular to the heterointerface. Assuming that the growth direction $z$ is along $\langle 001\rangle$, the InGaAs well layer is subjected to a biaxial compressive inplane strain parallel to $x$ along $\langle 100\rangle$ and to $y$ along $\langle 010\rangle$, and a uniaxial shear strain parallel to $z$ along $\langle 001\rangle$. The in-plane strain across the well will vary according to the composition of alloy concentration after interdiffusion. Hence, the biaxial in-plane strain and uniaxial shear strain after interdiffusion are given by

$$
\begin{aligned}
& \varepsilon_{x x}=\varepsilon_{y y}=\varepsilon\left(w_{1}, w_{2}\right) \\
& \varepsilon_{z z}=-2\left[c_{12}\left(w_{1}, w_{2}\right) / c_{11}\left(w_{1}, w_{2}\right)\right] \varepsilon\left(w_{1}, w_{2}\right) \\
& \varepsilon_{x y}=\varepsilon_{y z}=\varepsilon_{z x}=0
\end{aligned}
$$

where $\varepsilon\left(w_{1}, w_{2}\right)$ is the misfit factor between the well and the barrier such that it defines as negative for compressive strain, and $c_{i j}\left(w_{1}, w_{2}\right)$ are the elastic stiffness constants. The change in the bulk bandgap, $S_{\perp}\left(w_{1}, w_{2}\right)$, due to the hydrostatic component of strain is given by [23]

$$
\begin{aligned}
S_{\perp}\left(w_{1}, w_{2}\right)= & -2 a\left(w_{1}, w_{2}\right)\left[1-c_{12}\left(w_{1}, w_{2}\right) / c_{11}\left(w_{1}, w_{2}\right)\right] \\
& \cdot \varepsilon\left(w_{1}, w_{2}\right)
\end{aligned}
$$

where $a\left(w_{1}, w_{2}\right)$ is the hydrostatic deformation potential calculated from [23]

$$
\begin{aligned}
a\left(w_{1}, w_{2}\right)= & -\frac{1}{3}\left[c_{11}\left(w_{1}, w_{2}\right)+2 c_{12}\left(w_{1}, w_{2}\right)\right] \\
& \cdot \frac{d E_{g}\left(w_{1}, w_{2}\right)}{d P}
\end{aligned}
$$

where $d E_{g} / d P$ is the hydrostatic pressure coefficient of the lowest direct energy gap $E_{g}$. The splitting energy, $S_{/ /}\left(w_{1}, w_{2}\right)$, between the HH and LH band edges induced by the shear component of strain is given by

$$
\begin{aligned}
S_{/ /}\left(w_{1}, w_{2}\right)= & -b\left(w_{1}, w_{2}\right)\left[1+2 c_{12}\left(w_{1}, w_{2}\right) / c_{11}\left(w_{1}, w_{2}\right)\right] \\
& \cdot \varepsilon\left(w_{1}, w_{2}\right)
\end{aligned}
$$

where $b\left(w_{1}, w_{2}\right)$ is the shear deformation potential. The coupling between the LH and split-off band gives rise to asymmetric heavy-hole to light hole splitting [24], so that

$$
\begin{aligned}
S_{/ / \mathrm{HH}}\left(w_{1}, w_{2}\right)= & S / /\left(w_{1}, w_{2}\right) \\
S_{/ / \mathrm{LH}}\left(w_{1}, w_{2}\right)= & -1 / 2\left[S_{/ /}\left(w_{1}, w_{2}\right)+\Delta_{o}\left(w_{1}, w_{2}\right)\right] \\
& +1 / 2\left[9\left\{S_{/ /}\left(w_{1}, w_{2}\right)\right\}^{2}+\left\{\Delta_{o}\left(w_{1}, w_{2}\right)\right\}^{2}\right. \\
& \left.\quad-2 S_{/ /}\left(w_{1}, w_{2}\right) \Delta_{o}\left(w_{1}, w_{2}\right)\right]^{1 / 2}(9)
\end{aligned}
$$

where $\Delta_{o}\left(w_{1}, w_{2}\right)$ is the spin-orbit splitting.

The QW confinement potential after the intermixing process, $U_{r}\left(w_{1}, w_{2}\right)$, is obtained by modifying the unstrained potential profile after processing, $\Delta E_{r}\left(w_{1}, w_{2}\right)$, by the variable strain effects and is given by

$$
U_{r}\left(w_{1}, w_{2}\right)=\Delta E_{r}\left(w_{1}, w_{2}\right)-S_{\perp r}\left(w_{1}, w_{2}\right) \pm S_{\| r}\left(w_{1}, w_{2}\right)
$$

where $S_{\perp r}\left(w_{1}, w_{2}\right)=Q_{r} S_{\perp}\left(w_{1}, w_{2}\right)$, the + and - signs represent the confined $\mathrm{HH}$ and $\mathrm{LH}$ profiles, respectively, and $S_{\| c}\left(w_{1}, w_{2}\right)=0$.

\section{Band Structure}

To calculate the electron and hole wave function in QW, the multiband effective mass theory is applied. For most III-V semiconductors such as GaAs-based materials, it is a good approximation that the conduction and valence bands are decoupled. A parabolic bands model and Luttinger-Kohn Hamiltonian with strain components are used for the conduction and valence bands, respectively. The electron states near the conduction subband edge are assumed to be almost purely $s$-like and nondegenerate (excluding spin), while the hole states near the valence subband edge are almost purely $p$-like and four-fold degenerate (including spin). The envelope function scheme is adopted to describe the slowly varying (spatially extended) part of the wavefunction.

The wavefunctions of the electron and hole subband edge at the zone center of $\Gamma_{6}$ valley symmetry can be calculated separately, using the Ben-Daniel and Duke model [25] by the one-dimensional Schrodinger-like equation, which is written as follows:

$$
-\frac{\hbar^{2}}{2} \frac{d}{d z}\left[\frac{1}{m_{r}^{*}(z)} \frac{d \psi_{r \ell}(z)}{d z}\right]+U_{r}(z) \cdot \psi_{r \ell}(z)=E_{r \ell} \psi_{r \ell}(z)
$$

where $\psi_{r \ell}(z)$ is the wavefunction of the $\ell$ th subband for electrons $(r=c \ell)$ or holes $(r=v \ell)$, respectively, $m_{r}^{*}(z)$ is the corresponding carrier effective mass in the $z$ direction, and $E_{r \ell}$ is the subband-edge energy. Equation (11) is solved numerically using a finite difference method with the above confinement profile.

For the valence band structure, it is necessary to diagonalize the Luttinger-Kohn Hamiltonian with appropriate confinement potentials for heavy and light holes. The envelope function at finite $k_{/ /}$depends on $k_{/ /}$as a result of the mixing of the heavy and light hole bands. In this paper, the effective Hamiltonian approach described in [26] to calculate the valence subband structure is used. In this approach, the hole envelope function 
$\psi_{v \ell}\left(k_{/ /}, z\right)$ at any finite $k_{/ /}$not too far away from the zone center $\left(k_{/ /} \neq 0\right)$ can be expressed as a linear combination of the envelope functions $\psi_{v \ell}(z)$ at $k_{/ /}=0$ as follows:

$$
\psi\left(k_{\|}, z\right)=\sum_{\ell=1}^{N} \sum_{v=-3 / 2}^{3 / 2} d_{V, \ell}\left(k_{\|}\right) \psi_{V, \ell}(z)
$$

where $\psi_{v \ell}(z)$ is obtained by solving (11). This approximation is accurate within a limited range of $k / /$, as only a finite basis set is used in the linear expansion. A basis set of about 40 envelop functions is used in the present calculation and the results obtained are accurate within the operation energy range of QW laser. The effective Hamiltonian obtained is (13), shown at the bottom of the page, where

$$
\begin{aligned}
C_{\ell \ell^{\prime}}= & \left(\frac{3}{4}\right)^{1 / 2} \frac{\hbar^{2}}{m_{o}} \gamma_{2}\left(k_{x}-i k_{y}\right)^{2} \\
& \cdot \int_{0}^{L_{f}} d z \psi_{-3 / 2, \ell}(z) \psi_{1 / 2, \ell^{\prime}}(z) \\
B_{\ell \ell^{\prime}}= & (3)^{1 / 2} \frac{\hbar^{2}}{m_{o}} \gamma_{2}\left(-k_{x}-i k_{y}\right)^{2} \\
& \cdot \int_{0}^{L_{f}} d z \psi_{3 / 2, \ell}(z) \frac{\partial}{\partial z} \psi_{-1 / 2, \ell^{\prime}}(z) \\
E_{ \pm 3 / 2, s s^{\prime}}= & \delta_{s s^{\prime}} E_{H s}-\frac{\hbar^{2}}{2 m_{\| \mathrm{H}}} k_{\|}^{2} \\
E_{ \pm 1 / 2, s s^{\prime}}= & \delta_{s s^{\prime}} E_{L s}-\frac{\hbar^{2}}{2 m_{\| \mathrm{L}}} k_{\|}^{2}
\end{aligned}
$$

where $\gamma_{2}$ is the Luttinger parameter.

\section{Optical Gain}

The propagation direction of the generated photon is parallel to the QW layers, and the gain spectrum is calculated by the density matrix approach. The gain spectrum due to the transition between the conduction subband $p$ and the valence subband $q$ is given by

$$
\begin{aligned}
g_{p q}(E)=\int d E \sum_{p, q} & {\left[\frac{2 \pi q^{2} \hbar}{(2 \pi)^{2} n \varepsilon_{0} c m_{0}^{2} L_{z} E^{\prime}}\right.} \\
& \cdot \int d k_{/ /}\left|\hat{e} \cdot P_{p q}\left(k_{/ /}\right)\right|^{2} \\
& \cdot \delta\left(E_{p}^{e}\left(k_{/ /}\right)-E_{q}^{h}\left(k_{/ /}\right)-E^{\prime}\right) \\
& \left.\cdot\left[f^{e}\left(E_{p}^{e}\left(k_{/ /}\right)\right)-f^{h}\left(E_{q}^{h}\left(k_{/ /}\right)\right)\right]\right] \\
& \cdot \mathcal{L}\left(E-E^{\prime}\right)
\end{aligned}
$$

where $q$ is the electric charge, $n$ is the refractive index, $\varepsilon_{0}$ is the dielectric constant of the vacuum, $c$ is the speed of light, $L_{z}$ is the width of the quantum well, $E$ is the photon energy, $P_{p q}$ is the optical matrix element, $\hat{e}$ is a unit vector along the polarization direction of the optical electric field, and $f^{e}$ and $f^{h}$ are the Fermi distribution function for electron and hole in the conduction and valence subband, respectively. The spectral broadening of each transition is included, and the optical gain in a single QW structure is obtained with some spectral lineshape function over all transition energies $E^{\prime} . \mathcal{L}\left(E-E^{\prime}\right)$ is a Lorentzian line-broadening function and is given by

$$
\mathcal{L}\left(E-E^{\prime}\right)=\frac{1}{\pi} \frac{\hbar / \tau_{\text {in }}}{\left(E^{\prime}-E\right)^{2}+\left(\hbar / \tau_{\text {in }}\right)^{2}}
$$

where $\tau_{\text {in }}$ is the intraband relaxation time.

\section{RESUlTS AND Discussion}

The as-grown structure analyzed here is an $\mathrm{In}_{0.53} \mathrm{Ga}_{0.47} \mathrm{As}-\mathrm{In}_{0.52} \mathrm{Al}_{0.48} \mathrm{As}$ single quantum well, with as-grown well width $L_{z}=60 \AA$. The as-grown QW is assumed to be fabricated on InP substrate with InGaAs in the well and InAlAs in the barrier forming a lattice-matched QW structure.

The interdiffusion of the three cations (In, $\mathrm{Al}$, and $\mathrm{Ga}$ ) are assumed to obey Fick's second law, and the extent of interdiffusion is represented by the annealing time $t$ ranging from 0 to $10 \mathrm{~h}$ at a prescribed temperature of $812{ }^{\circ} \mathrm{C}$. The diffusion coefficients are obtained by fitting the diffusion model to the measured experimental data and their values were $D_{\mathrm{In}-\mathrm{In}}=2.98, D_{\mathrm{In}-\mathrm{Ga}}=-4.01, D_{\mathrm{Ga}-\mathrm{In}}=0.18$, and $D_{\mathrm{Ga}-\mathrm{Ga}}=6.69$ (in units of $10^{-18} \mathrm{~cm}^{2} \cdot \mathrm{s}^{-1}$ ) [22]. In the initial stage of interdiffusion, the indium atoms in the barrier diffused into the InGaAs well forming a slightly higher indium concentration. The variation of In, Al, and Ga composition profile in the interdiffused QW with annealing time is shown in Fig. 1(a)-(c). As InAs has a greater lattice constant than those of GaAs and AlAs, compressive strain arises inside the well with increased In content. Likewise, as Ga has diffused out to the barrier, a tensile strain is built up there near the heterointerface. Fig. 2 shows the type of induced strain along the growth axis for a range of different extents of intermixing represented by diffusion time. As can be seen, the positive sign (tensile) strain is developed in the barrier while the negative sign (compressive) strain in the well. As a result of the nonuniform distribution of the In, Al, and $\mathrm{Ga}$ atoms along the $z$ direction, the strain changes from zero in the outer barrier to a maximum positive value in the interface and then to a minimum value in the center of the well. As $t$ reaches $0.5 \mathrm{~h}$, two In concentration profile local peaks can be found at around $z= \pm 20 \AA$ [see Fig. 1(a)], this gives rise to the double peaked magnitude compressive stress in the well (see Fig. 2). As interdiffusion proceeds further, intermixing has reached the

$$
\left(\begin{array}{cccc}
E_{3 / 2}+S_{/ / \mathrm{HH}} & C & B & 0 \\
C^{*} & E_{-1 / 2}-S_{/ / \mathrm{LH}} & 0 & B^{T} \\
B^{*} & 0 & E_{+1 / 2}-S_{/ / \mathrm{LH}} & C^{T} \\
0 & B^{*} & C^{*} & E_{-3 / 2}+S_{/ / \mathrm{HH}}
\end{array}\right)
$$




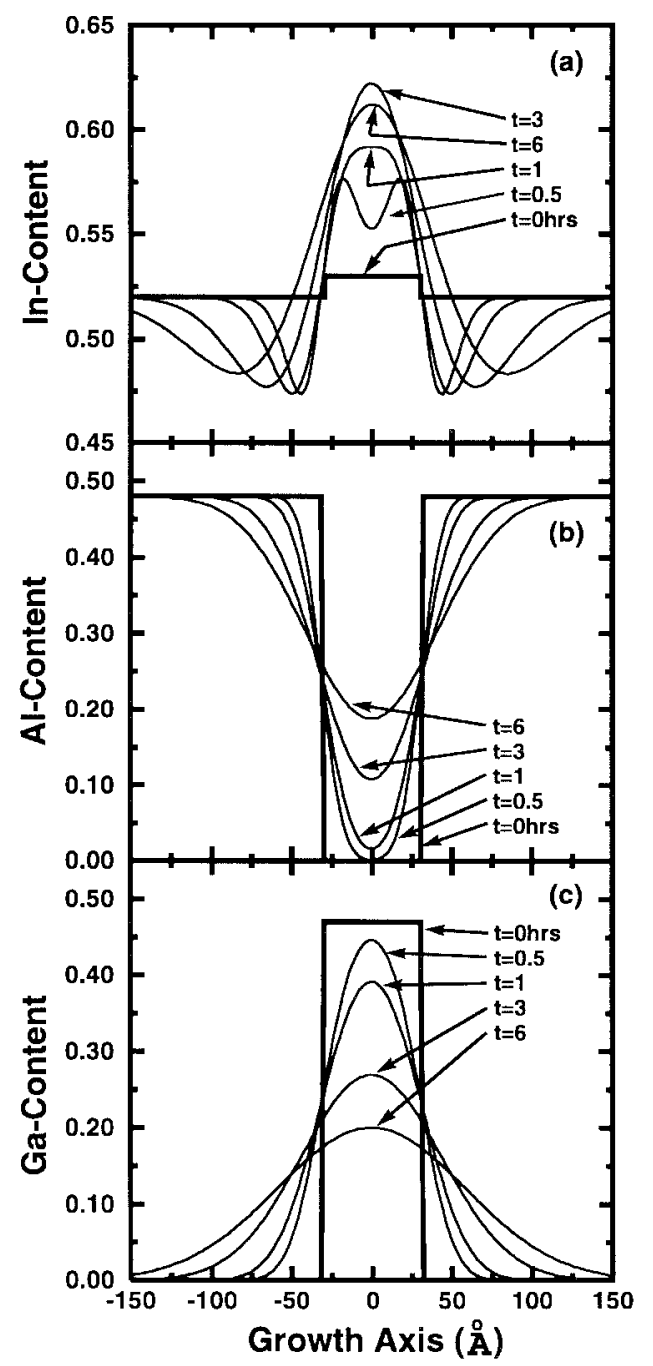

Fig. 1. Three cations compositional profiles of $\operatorname{In}_{0.53} \mathrm{Ga}_{0.47} \mathrm{As}-$ $\mathrm{In}_{0.52} \mathrm{Al}_{0.48} \mathrm{As} \mathrm{QW}$ with as-grown well width of $60 \AA$ at $812{ }^{\circ} \mathrm{C}$ annealing temperature for as-grown $(t=0)$ and several annealing times.

center of the well and the compressive strain has now increased in magnitude at the well center while the tensile strain also spreads out more into the barriers. The effective thickness of the InGaAs well should therefore be monitored in order to avoid dislocation. Maximum In concentration of 0.62 is obtained in the center of well as $t$ reaches $3 \mathrm{~h}$, which results in a corresponding $0.64 \%$ compressive strain. The reason to have such a high concentration of In in the well layers is due to a much higher $\mathrm{Ga}$ diffusion rate so that it takes up all the vacancy in the barrier and thus suppresses the In diffusion out to the barrier [22]. For annealing time exceeding $3 \mathrm{~h}$, the compressive strain relaxes in a gradual manner, as shown in Fig. 3. For longer annealing time $(t>6 \mathrm{~h})$, all three interdiffused composition profiles become evenly distributed. This gives rise to a reduction of strain in the well and barrier and thus relaxes the stress, as the constitution of the QW is now approaching that of a uniform InAlGaAs bulk material.

After Al diffused into the InGaAs well, InAlGaAs is formed with a larger bulk bandgap energy; this decreases the band offset as well as the depth of the electron and hole potential profiles. The hydrostatic and shear components of the com-

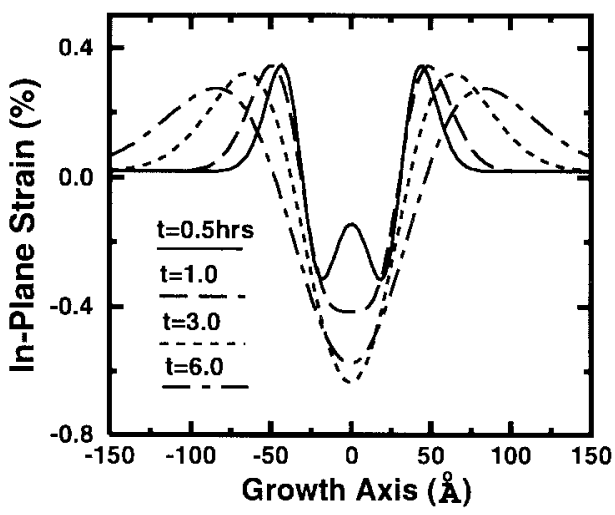

Fig. 2. In-plane strain across the interdiffused QW for various annealing times, $t=0.5 \mathrm{~h}$ (solid line), $t=1 \mathrm{~h}$ (long dashed line), $t=3 \mathrm{~h}$ (dashed line), and $t=6 \mathrm{~h}$ (dotted-dash line)

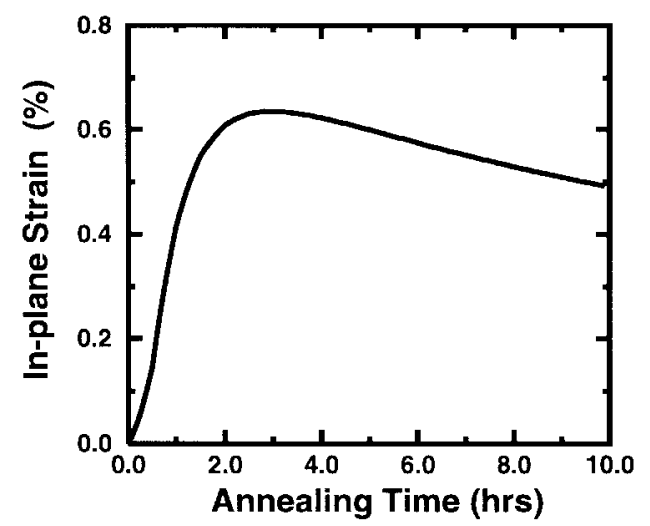

Fig. 3. The in-plane compressive strain at the well center for various annealing times.

pressive strain modify the depth of the valence potential in different manners [27]. The shear component of the compressive strain induces the HH-LH band edge splitting $S_{\|}$, which increases the depth of the heavy hole potential while it decreases the depth of the light hole one. For $t=0-1.5 \mathrm{~h}$, the Al content inside the well is still low and this causes a small increase in the bandgap energy of the InAlGaAs well. As the effect of compressive strain is more pronounced in this range, the depth of the heavy hole well is deepened while that of the light hole is lessened. The maximum depth of the heavy hole potential is $0.24 \mathrm{eV}$, which occurs at $t$ between 1 and $1.5 \mathrm{~h}$. As annealing time goes beyond $1.5 \mathrm{~h}$, reduction in band offset due to Al diffusion becomes much more pronounced in the well, which results in a gradual reduction in the depth of both the heavy hole and light hole potential wells. A similar trend can be observed in the conduction potential.

In the as-grown QW, the ground-state heavy hole (HH1) is the highest subband followed by the ground-state light hole (LH1) with an electron-like effective mass at the zone center. Their energy separation $\Delta E_{\mathrm{SUB}}$ [28], after interdiffusion, is affected by the compressive strain. This separation is an interesting parameter to explore in order to explain the magnitude of optical gain. The $\Delta E_{\mathrm{SUB}}$ is not taken here exactly at the zone center but at a nearby point in order to avoid the strong mixing. In general, $\Delta E_{\mathrm{SUB}}$ is mainly affected by the depth and width of the potential well and the 


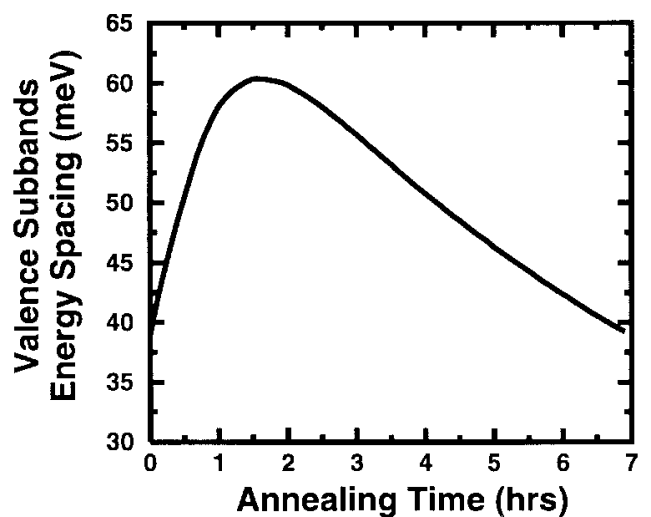

Fig. 4. The valence band energy separation between the HH1 and LH1 states at subband edge for various values of annealing time.

effect of strain. In the case of compressive strain, $\Delta E_{\mathrm{SUB}}$ increases with the depth of the $\mathrm{HH}$ potential and decreases with well width. A variation of $\Delta E_{\mathrm{SUB}}$ against diffusion time is plotted in Fig. 4. $\Delta E_{\mathrm{SUB}}$ attains a maximum of $60 \mathrm{meV}$ around an annealing time $t=1.5 \mathrm{~h}$ as a consequence of the rising compressive strain and a small effective well width. In the lattice-matched as-grown well, no strain is induced in a relatively narrow well width, hence a small $\Delta E_{\mathrm{SUB}}$ (39 $\mathrm{meV}$ ) is expected. The increase of $\Delta E_{\mathrm{SUB}}$ from $39 \mathrm{meV}$ of the as-grown QW to $60 \mathrm{meV}$ of an 1.5-h annealed QW is mainly due to the compressive strain created in the well. For annealing time exceeding $1.5 \mathrm{~h}$, as the depth of the $\mathrm{HH}$ and LH potentials both decrease gradually and the well becomes wider, subbands are densely packed and the $\Delta E_{\text {SUB }}$ starts to decrease. The subband energy spacing has a notable effect on the effective mass of the heavy and light holes. As $\Delta E_{\mathrm{SUB}}$ increases, the interaction between these two bands reduces and so parabolicity can be restored. Reduction in the effective mass of the heavy hole can be observed for in-plane wavevector $k \geq 0.02$. Consequently, a reduction in the averaged density of states (DOS) is expected. Fig. 5 shows the top four valence subbands structures of the DFQW's. In the initial stage of the intermixing process, the average DOS reduces with a rapid rate. In the latter stage of intermixing, strong mixing among these valence subbands is expected to be observed and the averaged DOS increases gradually with a low rate. These subbands become densely packed as intermixing proceeds further and in the ultimate limit, it results with a bulk-like material characteristics.

Optical gain is mainly dependent on the joint DOS, Fermi occupation factor, and optical matrix element [28]. The TE mode optical gain spectra of the DFQW with various extent of intermixing (indicated by diffusion time $t$ ) are showed in Fig. 6. The carrier density used in this calculation is fixed at $1.2 \times 10^{12} \mathrm{~cm}^{-2}$. In this figure, the peak gain energy can be seen to shift toward higher energies from $\lambda=1.47 \mu \mathrm{m}$ $(0.842 \mathrm{eV})$ of an as-grown structure all the way to $\lambda=$ $1.22 \mu \mathrm{m}(1.016 \mathrm{eV})$ of a DFQW $(t=6 \mathrm{~h})$. In the initial stage of interdiffusion (i.e., $t \leq 1.5 \mathrm{~h}$ ), an enhancement in peak gain magnitude up to $40 \%$ can be observed at $t=$ $1.0 \mathrm{~h}$. This is because, as interdiffusion reaches this stage, a large $\Delta E_{\mathrm{SUB}}=58 \mathrm{meV}$ (see Fig. 4) and a reduction in (a)

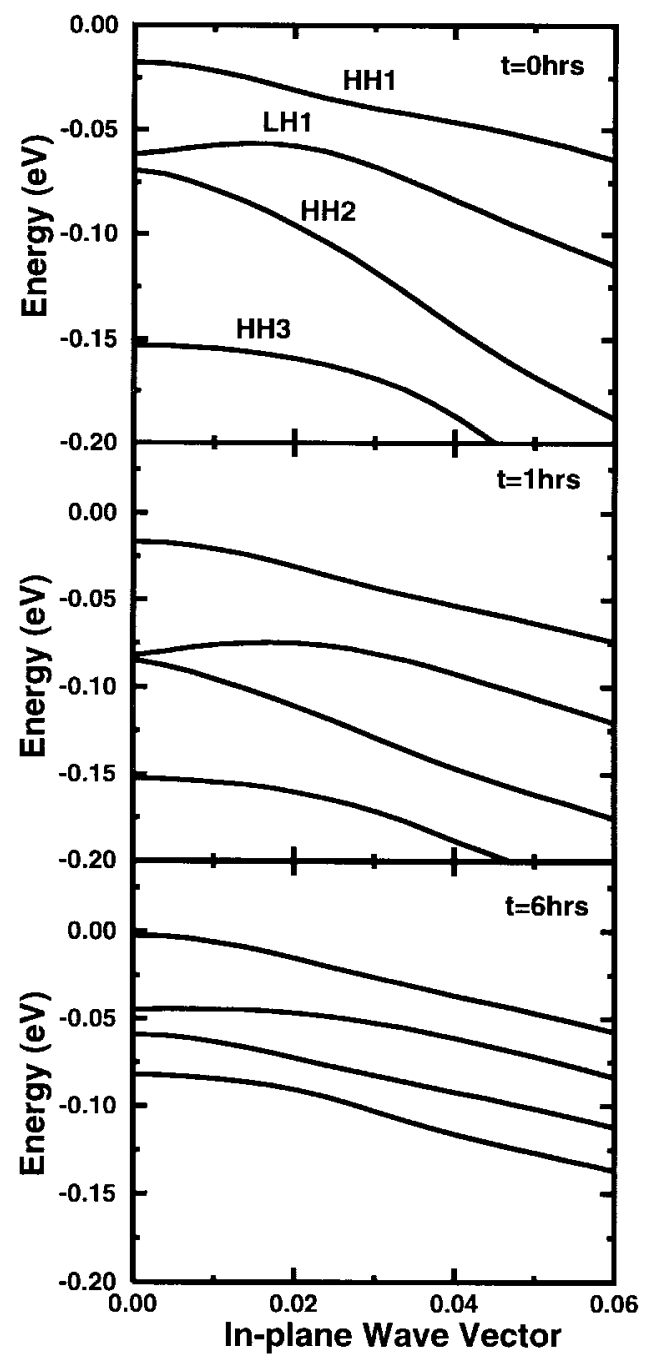

Fig. 5. The valence subbands dispersion as a function of the in-plane wave vector for various annealing time. (a) $t=0 \mathrm{~h}$. (b) $t=1 \mathrm{~h}$. (c) $t=6 \mathrm{~h}$

the DOS for the compressively strained DFQW results, which give rise to an improvement of the Fermi occupation factor. As the Fermi occupation factor, $f^{e}\left(E_{p}^{e}\left(k_{/ /}\right)\right)-f^{h}\left(E_{q}^{h}\left(k_{/ /}\right)\right)$, has a dominant role in the gain spectrum of the compressively strained QW, a large enhancement in optical gain is obtained. In the case of the as-grown $\mathrm{QW}$, as it has a small $\Delta E_{\mathrm{SUB}}$ and a large DOS for its unstrained well, which results in a relatively lower optical gain. Thus, by employing QW intermixing, laser performance of InGaAs-InAlAs QW can be greatly enhanced. The peak gain magnitude will decrease as the extent of interdiffusion gets larger, since the QW structure is becoming more and more bulk-like.

The peak wavelength of the gain spectrum is plotted in Fig. 7 in order to determine the best performance of the DFQW laser by the intermixing technique. The peak wavelength of the gain spectrum for InGaAs-InAlAs is quite insensitive to the variation of the injected carrier density. The shift of the peak wavelength for different cases of annealing times are less than $0.025 \mathrm{eV}$ over a range of $1.0-2.5 \times 10^{12} \mathrm{~cm}^{-2}$.

Interpolating the peak gain as a function of carrier density, the transparency carrier density for different annealing times 


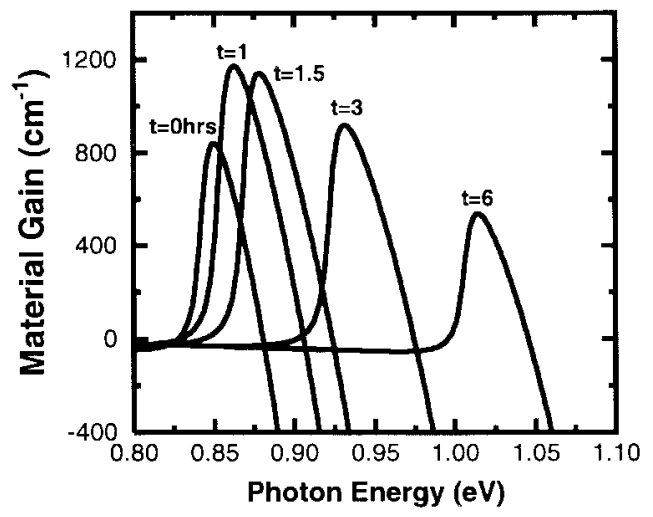

Fig. 6. Optical gain spectra of $\mathrm{In}_{0.53} \mathrm{Ga}_{0.47} \mathrm{As}-\mathrm{In}_{0.52} \mathrm{Al}_{0.48} \mathrm{As} \mathrm{QW}$ with as-grown well width of $60 \AA$ and at a fixed carrier injection level of 1.2 $\times 10^{12} \mathrm{~cm}^{-2}$ for various annealing times.

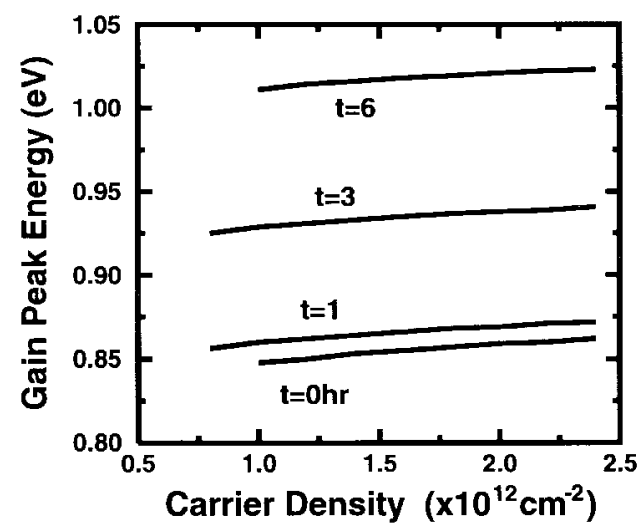

Fig. 7. The variation of gain peak energy as a function of carrier density for various annealing times.

of the DFQW can be obtained. At a particular annealing time and carrier injection level $N$, the optical gain $G(N, t)$ is found to have a simple expression [29]

$$
G(N, t)=a_{o}(t) \ln \left(\frac{N}{N_{o}(t)}\right)
$$

where the gain coefficient $a_{o}(t)$ is a fitted parameter and $N_{o}(t)$ is the transparency carrier density. All these parameters are a function of the annealing time. The gain coefficient and transparency carrier density can be empirically expressed as a function of the annealing time (in hours)

$$
\begin{aligned}
a_{o}(t)= & \left(12 t^{2}+251 t-2580, \quad\left[\mathrm{~cm}^{-1}\right]\right. \\
N_{o}(t)= & 2.3 \times 10^{-4} t^{4}-5.7 \times 10^{-3} t_{3}+4.9 \times 10^{-2} t^{2} \\
& -0.14 t+0.85 \quad\left[\times 10^{12} \mathrm{~cm}^{-2}\right] .
\end{aligned}
$$

The linear gain saturation effects are taken into consideration here. For simplicity, the nonlinear gain coefficient has been neglected.

In analyzing the effect of intermixing through various annealing times, we plot the optical gain peak as a function of the injection carrier density, as shown Fig. 8. The optical gain during the initial stages of intermixing, such as at an annealing time $t=1 \mathrm{~h}$, is always larger than the as-grown $\mathrm{QW}$ for all carrier density considered. For the annealing time of $3 \mathrm{~h}$, the optical gain has been reduced although it is still greater than that of the as-grown case except at large carrier density. As the process of intermixing becomes more extensive, such as

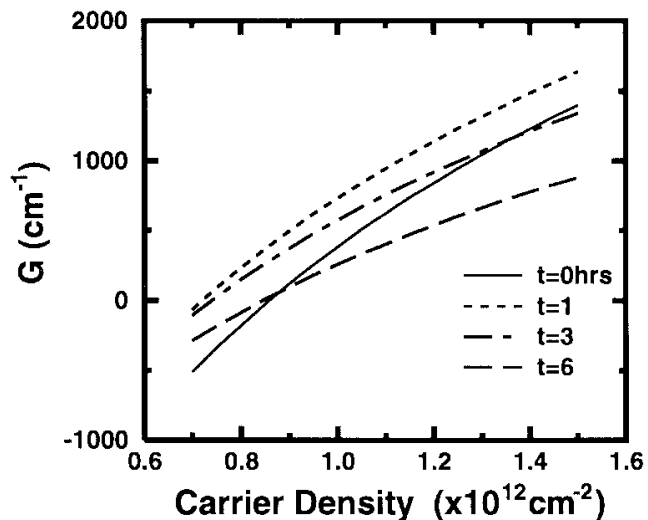

Fig. 8. The optical gain $(G)$ in (16) as a function of carrier density $(N)$ for various annealing times.

$t=6 \mathrm{~h}$, the optical gain is now lower than the as-grown QW for most carrier densities except at the lower level end, which is absorption. For the best operating performance of DFQW lasers, considering a small injected carrier density and a large optical gain, a level of $1.2 \times 10^{12} \mathrm{~cm}^{-2}$ injected carrier density should be used and this condition can be obtained at around $1 \mathrm{~h}$ annealing time.

In the present model, the material differential gain is defined as $\partial g / \partial N$. The differential gain, which is defined as the rate of increase of optical gain with injected carrier density, is also modifiable by interdiffusion. It depends on the magnitude of the optical gain, well width, and the subband energy spacing $\Delta E_{\mathrm{SUB}}$ in the compressively strained well. For $t \leq 1 \mathrm{~h}$, subband energy spacing $\Delta E_{\mathrm{SUB}}$ increases while the effective well width decreases, both of which contribute to an increase in differential gain. However, these are compensated by an increase in magnitude of the optical gain which results in a reduction of the differential gain. Thus, a small variation of differential gain can be observed during this initial period of annealing. As interdiffusion proceeds further, reduction in $\Delta E_{\mathrm{SUB}}$ and increase in effective well width are more pronounced than the reduction in optical gain, thus a gradual drop of differential gain is observed. The relationship between differential gain and annealing time is plotted in Fig. 9. As shown, high differential gain is shown to maintain for $t \leq 1 \mathrm{~h}$ with a value of $0.22 \times 10^{-9} \mathrm{~cm}^{-1}$. As interdiffusion proceeds, the differential gain drops gradually between $t=2$ and $7 \mathrm{~h}$ to the values of $0.19 \times 10^{-9} \mathrm{~cm}^{-1}$ (17\% reduction) and $0.13 \times$ $10^{-9} \mathrm{~cm}^{-1}$ (42\% reduction), respectively. This latter results in an unsatisfactory performance, as large increases in threshold carrier density and reduction in modulation bandwidth are expected for laser operations.

\section{CONCLUSION}

We have presented the multiple cations interdiffusion of $\mathrm{In}_{0.53} \mathrm{Ga}_{0.47} \mathrm{As}-\mathrm{In}_{0.52} \mathrm{Al}_{0.48} \mathrm{As}$ single $\mathrm{QW}$ with well width of $60 \AA$. The suppression of indium sublattice movement in the well exerts a significant influence on the compressive strain. At an annealing time of $t=3 \mathrm{~h}$, a maximum In concentration of 0.62 is found in the center of the well which results in a compressive strain with $0.64 \%$ misfit. This creation of strain in the well through interdiffusion results in an enhancement of the 


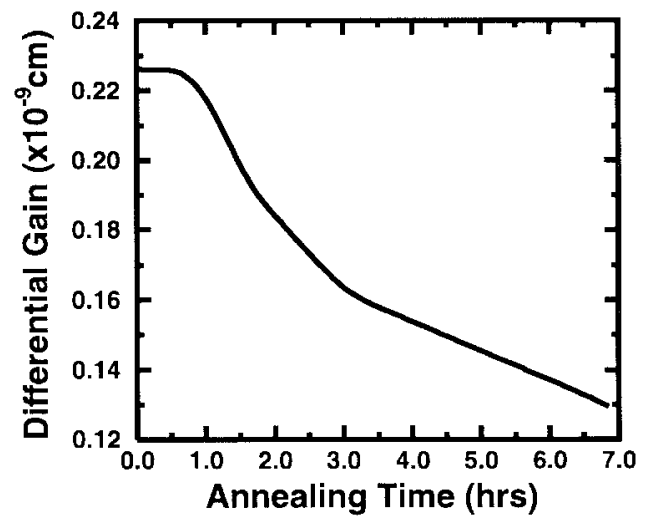

Fig. 9. The differential gain of the $\mathrm{In}_{0.53} \mathrm{Ga}_{0.47} \mathrm{As}-\mathrm{In}_{0.52} \mathrm{Al}_{0.48} \mathrm{As} \mathrm{QW}$ as a function of annealing time.

optical gain by $40 \%$ as compared with the as-grown unstrained wells. This is because the compressive shear component of strain results in a reduced effective well width and a deepened potential well, thus, contributing to the splitting of the heavy and light holes and resulting in an increase of the subband energy spacing. A large subband energy spacing of $58 \mathrm{meV}$ can be obtained at an annealing time of $1.0 \mathrm{~h}$, which is responsible for the substantial optical gain enhancement. The interdiffusion of the QW produce a blue shift of the peak gain energy, shifting from $0.842 \mathrm{eV}(\lambda=1.47 \mu \mathrm{m})$ to $1.016 \mathrm{eV}(\lambda=$ $1.22 \mu \mathrm{m})$, which can be useful in bandgap tuning applications. Interdiffusion in the $\mathrm{In}_{0.52} \mathrm{Al}_{0.48} \mathrm{As}-\mathrm{In}_{0.53} \mathrm{Ga}_{0.47} \mathrm{As}$ structure with initial annealing time between 1 and $1.5 \mathrm{~h}$ will give the best laser operation, in terms of threshold current, optical gain, and satisfactory differential gain.

\section{REFERENCES}

[1] E. H. Li, Ed., Quantum Well Intermixing for Photonics, Milestone Series. Bellingham, WA: SPIE, 19978 vol. 145.

[2] K. Wakita, I. Kotaka, O. Mitomi, H. Asai, and Y. Kawamura, "Observation of low-chirp modulation in InGaAs-InAlAs multiple-quantum well optical modulators inder $30 \mathrm{GHz}$," IEEE Photon. Technol. Lett., vol. 3, pp. 138-140, 1991

[3] T. Ido, H. Sano, D. J. Moss, S. Tanaka, and A. Takai, "Strained InGaAs/InAlAs MQW electro-absorption modulators with large bandwidth and low driving VolHage," IEEE Photon. Technol. Lett., vol. 6, pp. 1207-1209, 1994.

[4] H. W. Wan, T. C. Chong, and S. J. Chua, "Considerations for polarization insensitive optical switching and modulation using strained InGaAs/InAlAs quantum well structure," IEEE Photon. Technol. Lett., vol. 3, pp. 730-732, 1991.

[5] J. E. Zucker, K. L. Jones, G. R. Jacobovitz, B. Tell, K. Brown-Gobeler, T. Y. Chang, N. J. Sauer, M. D. Divino, M. Wegener, and D. S. Chemla, "InGaAs-InAlAs quantum well intersecting waveguide switch operating at $1.55 \mu \mathrm{m}$," IEEE Photon. Technol. Lett., vol. 2, pp. 804-806, 1990.

[6] E. H. Li, B. L. Weiss, and K. S. Chan, "Effect of interdiffusion on the subbands in an $\mathrm{Al}_{x} \mathrm{Ga}_{1-x} \mathrm{As} / \mathrm{GaAs}$ single- quantum-well structure," Phys. Rev. B, vol. 46, pp. 15181-15192, 1992.

[7] W. C. Shiu, J. Micallef, I. Ng, and E. H. Li, "Effects of different cation and anion interdiffusion rates in disordered $\mathrm{InGaAs} / \mathrm{InP}$ single quantum wells", Jpn. J. Appl. Phys. (Part 1), vol. 34, no. 4, pp. 1778-1783, 1995.

[8] J. C. P. Chang, J. M. Woodall, M. R. Melloch, I. Lahiri, D. D. Nolte, N. Y. Li, and C. W. Tu, "Investigation of interface intermixing and roughening low-temperature-grown $\mathrm{AlAs} / \mathrm{GaAs}$ multiple quantum wells during thermal annealing by chemical lattice imaging and X-ray diffraction," Appl. Phys. Lett., vol. 67, pp. 3491-3493, 1995.

[9] W. J. Taylor, N. Kuwata, I. Yoshida, T. Katsuyama, and H. Hayashi, "Interdiffusion of GaAs/ $\mathrm{Ga}_{1-x} \mathrm{In}_{x}$ As quantum wells," J. Appl. Phys., vol. 73, pp. 8653-8655, 1993.
[10] J. Micallef, E. H. Li, and B. L. Weiss, "Exciton optical absorption in disordered, strained InGaAs/GaAs single quantum wells," Supperlattices Microstruct., vol. 13, pp. 315-321, 1993.

[11] S. S. Rao, W. P. Gillin, and K. P. Homewood, "Interdiffusion of the group-III sublattice in In-Ga-As-P/In-Ga-As-P and In-Ga-As heterosturctures," Phys. Rev. B, vol. 50, pp. 8071-8073, 1994.

[12] K. Mukai, M. Sugawara, and S. Yamazaki, "Interdiffusion process in lattice-matched $\operatorname{In}_{x} \mathrm{Ga}_{1-x} \mathrm{As}_{y} \mathrm{P}_{1-y} / \mathrm{InP}$ and $\mathrm{GaAs} / \mathrm{Al}_{x} \mathrm{Ga}_{1-x} \mathrm{As}$ quantum wells," Phys. Rev. B, vol. 50, pp. 2273-2282, 1994.

[13] R. Schorer, E. Friess, K. Eberl, and G. Abstreiter, "Structural stability of short-period $\mathrm{Si} / \mathrm{Ge}$ superlattices studied with Raman spectroscopy," Phys. Rev. B, vol. 44, pp. 1772-1781, 1991.

[14] K. P. Homewood, W. P. Gillin, R. E. Pritchard, W. S. Truscott, and K. E. Singer, "Thermal processing of $\mathrm{GaAsSb} / \mathrm{GaAs}$ low-dimensional strained-layer structures," Superlattices Microstruc., vol. 7, pp. 359-361, 1990.

[15] A. Rosenauer, T. Reisinger, E. Steinkirchner, J. Zweck, and W. Gebhardt, "High resolution transmission electron microscopy determination of Cd diffusion in CdSe/ZnSe single quantum well structures," J. Cryst. Growth, vol. 152, pp. 42-50, 1995.

[16] D. G. Deppe and N. Holonyak, Jr., "Atom diffusion and impurityinduced layer disordering in quantum well III-V semiconductor heterostructure," J. Appl. Phys., vol. 64, pp. R93-R113, 1988.

[17] I. Harrison, "Impurity-induced disordering in III-V multi-quantum well and superlattices," J. Mater. Sci., vol. 4, pp. 1-28, 1993.

[18] J. H. Marsh, "Quantum well intermixing," Semicond. Sci. Technol., vol. 8, pp. 1136-1155, 1993.

[19] S. Burkner, J. D. Ralston, S. Weisser, J. Rosenzweig, E. C. Larkins, R. E. Sah, and J. Fleissner "Wavelength tuning of high-speed InGaAs-GaAsAlGaAs pseudomorphic MQW lasers via impurity-free interdiffusion," IEEE Photon. Technol. Lett., vol. 7, pp. 941-943, 1995.

[20] W. C. H. Choy and E. H. Li, "The application of an interdiffused quantum well in a normally on electroabsorptive Fabry-Perot reflection modulator," IEEE J. Quantum Electron., vol. 33, pp. 382-392, 1997.

[21] E. S. Koteles, S. Charbonneau, P. Poole, J. J. He, M. Davies, M. Dion, G. Aers, Y. Feng, I. V. Mitchell, and R. D. Goldberg, "Photonic integration using quantum well shape modification," Phys. Canada, pp. 251-255, Sept./Oct. 1996.

[22] Y. Chan, W. C. Shiu, W. K. Tsui, and E. H. Li, "Multiple cations interdiffusion in $\operatorname{In}_{0.53} \mathrm{Ga}_{0.47} \mathrm{As} / \mathrm{In}_{0.52} \mathrm{Al}_{0.48}$ As quantum well," Proc. Mater. Res. Soc., vol. 450, pp. 337-382, 1997.

[23] H. Asai and K. Oe, "Energy band-gap shift with elastic strain in $\mathrm{Ga}_{x} \mathrm{In}_{1-x} \mathrm{O}$ optixial layers on (001) GaAs substrates," J. Appl. Phys., vol. 54, pp. 2052-2056, 1993

[24] R. People, "Indirect band gap of coherently strained $\mathrm{Ge}_{x} \mathrm{Si}_{1-x}$ bulk alloys on $\langle 001\rangle$ silicon substrates," Phys. Rev. B, vol. 32, pp. 1405-1408, 1985.

[25] D. J. Ben-Daniel and C. B. Duke, "Space-charge effects on electron tunneling," Phys. Rev., vol. 152, pp. 683-692, 1966.

[26] K. S. Chan, "The effects of the hole sub-band mixing on the energies and oscillator strengths of excitons in a quantum well," J. Phys. C, vol. 19, L125-L130, 1996.

[27] J. Micallef, E. H. Li, and B. L. Weiss, "The effect of interdiffusion on the subband-edge of $\mathrm{In}_{0.53} \mathrm{Ga}_{0.47} \mathrm{As} / \mathrm{InP}$ single quantum wells," $J$. Appl. Phys., vol. 73, pp. 7524-7532, 1993.

[28] S. Seki, T. Yamanaka, W. Lui, Y. Yoshikumi, and K. Yokoyama, "Theoretical analysis of pure effects of strain and quantum confinement on differential gain in InGaAsP/InP strained layer quantum-well lasers," IEEE J. Quantum Electron., vol. 30, pp. 500-510, 1994.

[29] J. Hong, W. P. Huang, and T. Makino, "Effect of linear gain saturation on small-signal dynamics of MQW DFB lasers," IEEE Photon. Technol. Lett., vol. 5, pp. 1373-1376, 1993.

Michael C. Y. Chan, photograph and biography not available at the time of publication.

Y. Chan, photograph and biography not available at the time of publication.

E. Herbert Li (S'87-M'88-SM'95), for biography, see p. 92 of the January 1998 issue of this JournaL. 